\title{
Dependência espacial da fertilidade do solo sob plantio direto e suas relações com a produtividade da soja
}

\author{
Ricardo Londero ${ }^{1}$, Rogério Barbosa de Oliveira ${ }^{2}$, Jackeline Matos do Nascimento ${ }^{3}$, Sálvio Napoleão Soares \\ Arcoverde $^{4}$, Mateus Luiz Secretti ${ }^{5}$
}

DOI: https://doi.org/10.35699/2447-6218.2020.25450

\begin{abstract}
Resumo
As ferramentas de agricultura de precisão permitem interpretação das causas de variações da fertilidade do solo e, assim, realizar intervenções de maneira localizada por meio da aplicação de insumos em taxa variável. Objetivou-se analisar a dependência espacial da fertilidade do solo e sua correlação com a produtividade da soja Glycine max (L) em Dourados - MS. Foi realizado o mapeamento com GPS de navegação global demarcando a área de 50 hectares, para posterior geração da malha de amostragem de solo e de produtividade. A partir da distribuição dos pontos, foi realizada a coleta do solo na camada de 0,00-0,20 m a cada 1 hectare, sendo as amostras identificadas e submetidas às análises químicas em laboratório, nas quais foram avaliados: CTC, H+Al, Fósforo, Potássio, Cálcio, Magnésio, pH, V\%, e Matéria Orgânica. Além de determinada a produtividade da cultura. Os dados foram submetidos à estatística descritiva, geoestatistica e correlação de Pearson. Todos os atributos químicos do solo encontraram-se em níveis adequados para produção da soja. Quanto à dependência espacial dos atributos químicos do solo e produtividade da soja, estes apresentaram forte dependência espacial, exceto Matéria Orgânica cuja dependência foi moderada. O semivariograma constatou que as variáveis $\mathrm{pH}$ e Matéria Orgânica foram ajustadas ao modelo esférico, Fósforo e $\mathrm{H}+\mathrm{Al}$ ao modelo exponencial e os demais ao modelo gaussiano. Com base nos coeficientes de correlação entre produtividade de soja e atributos químicos do solo foi constatado significância positiva para Fósforo $(0,30)$ e significância negativa para CTC $(-0,30)$ e $\mathrm{H}+\mathrm{Al}(-0,29)$.
\end{abstract}

Palavras-Chave: Geoestatística. Krigagem. Latossolos. Semivariogramas.

\section{Spatial dependence of soil fertility under no-tillage their relationship of yield soybean}

\begin{abstract}
:
Precision agricultural tools allow interpretation of the causes of variations in soil fertility and, thus, carry out interventions in a localized manner through the application of inputs at a variable rate. The objective was to analyze the spatial dependence on soil fertility and its correlation with the yield of soybean Glycine max (L) in Dourados - MS. Global navigation GPS mapping was carried out, demarcating the area of 50 hectares, for subsequent generation of the soil sampling and yield grid. From the distribution of the points, the soil was collected in the $0.00-0.20 \mathrm{~m}$ layer for each 1 hectare, and the samples were identified and submitted to chemical analysis in the laboratory, in which the
\end{abstract}

\footnotetext{
${ }^{1}$ Centro Universitário da Grande Dourados. Dourados, MS. Brasil. https://orcid.org/0000-0002-7039-2447

${ }^{2}$ Centro Universitário da Grande Dourados. Dourados, MS. Brasil. https://orcid.org/0000-0003-2332-663

${ }^{3}$ Centro Universitário da Grande Dourados. Dourados, MS. Brasil. https://orcid.org/0000-0001-5863-4450

${ }^{4}$ Universidade Federal da Grande Dourados. Dourados, MS. Brasil. https://orcid.org/0000-0002-0453-4566

${ }^{5}$ Centro Universitário da Grande Dourados. Dourados, MS. Brasil. https://orcid.org/0000-0001-9538-4104

*Autor para correspondência: salvionapoleao@gmail.com
}

Recebido para publicação em 03 de setembro de 2020. Aceito para publicação em 19 de novembro de 2020. e-ISSN: 2447-6218 / ISSN: 2447-6218. Atribuição CC BY. 
Londero, R. et al.

following were evaluated: CTC, $\mathrm{H}+\mathrm{Al}$, Phosphorus, Potassium, Calcium, Magnesium, pH, V\%, and Organic Matter. In addition to determining the yield of the crop. The data were submitted to descriptive statistics, geostatistics and Pearson correlation. All the chemical attributes of the soil were found at adequate levels for soybean yield. As for the spatial dependence on the chemical attributes of the soil and soybean productivity, these showed strong spatial dependence, except for Organic Matter, whose dependence was moderate. The semivariogram found that the variables $\mathrm{pH}$ and Organic Matter were adjusted to the spherical model, Phosphorus and $\mathrm{H}+\mathrm{Al}$ to the exponential model and the others to the Gaussian model. Based on the correlation coefficients between soybean yield and soil chemical attributes, a positive significance was found for Phosphorus (0.30) and a negative significance for CTC (-0.30) and $\mathrm{H}+\mathrm{Al}(-0.29)$.

Keywords: Geostatistics. Kriging. Oxisol. Semivariograms.

\section{Introdução}

A grande demanda no mercado internacional proporcionou rápida expansão da soja no Brasil, que ocorreu pela tomada de áreas cultivadas com outras culturas e, principalmente, da conquista de novas fronteiras agrícolas (Freitas; Mendonça, 2016). O produtor tem incrementado o uso de tecnologia a fim de aumentar a produtividade e, dessa forma, melhorar sua rentabilidade. Assim, a produtividade da soja passou de $2.823 \mathrm{~kg}$ ha-1 na safra 2006/07, para produção recorde $3.394 \mathrm{~kg}$ ha-1 na safra $2017 / 18$, um aumento de $20 \%$, já na safra 2018/19 a produção foi de $3.206 \mathrm{~kg}$ ha- 1 , a estimativa para este ano agrícola (2019/20) é de $3.292 \mathrm{~kg}$ ha-1 (CONAB, 2019).

Nos últimos 40 anos ocorreu significativa expansão das áreas destinadas à produção de grãos, fazendo-se necessário a adoção de tecnologias e ferramentas para melhorar o gerenciamento da propriedade rural (Trentin et al., 2018). Dentre os meios usados para elevar a produtividade está a agricultura de precisão (AP), que engloba um conjunto de ferramentas e tecnologias que permitem o gerenciamento agrícola baseado na variabilidade espacial e temporal da unidade produtiva, visando ao aumento de retorno econômico e à redução do impacto ao ambiente (Brasil, 2017).

Entre as ferramentas utilizadas ao longo do tempo, destacam-se o emprego de amostragem de solo em grade e a adubação a taxa variada a lanço (Soares Filho; Cunha, 2015), sobretudo para o gerenciamento de sistemas de produção intensivo, principalmente àqueles voltados à produção de grãos e algodão, cuja atividade gera um custo elevado especialmente com a adubação (Zonta et al., 2014).

Podem-se citar como as principais tecnologias ou operações agrícolas referidas como AP, os sistemas de guia e automação agrícola, manejo fitossanitário, amostragem de solo, sistemas de corretivos e fertilizantes em taxa variável, semeadura em taxa variável, mapeamento de produtividade e sensoriamento. A prática de AP mais disseminada no Brasil, atualmente, consiste da amostragem de solo georreferenciada para o mapeamento da fertilidade dos talhões de cultivo (Resende et al., 2014; Soares Filho; Cunha, 2015).
De acordo com Dalchiavon et al. (2012), a variabilidade dos atributos químicos do solo é consequência de interações complexas em processos de sua formação e de práticas de manejo do solo e da cultura, com impacto principalmente nas camadas superficiais do solo. O estudo da variabilidade espacial dos atributos da fertilidade do solo mostra-se uma importante ferramenta na compreensão da distribuição espacial dos macronutrientes, auxiliando no planejamento de coletas de amostras e nas práticas de manejo do solo (Zonta et al., 2014) e, portanto, no auxílio à tomada de decisão que vise atender à exigência nutricional da cultura (Santos et al., 2015).

Para se ter um melhor entendimento desta dinâmica do solo, é utilizada a geoestatística, que é uma ferramenta, que permite obter informações essenciais ao manejo agrícola a partir da variabilidade espacial de características do solo (Grego; Vieira, 2005; Santos et al., 2017). Com isso, a descrição da distribuição espacial dos macronutrientes, considerando-se os efeitos conjuntos de práticas de fertilização/correção e de manejo do solo, permite melhor entendimento do comportamento dos elementos no solo, gerando informações importantes como subsídio à melhoria da fertilidade do solo e à sustentabilidade dos sistemas de produção agrícola.

Portanto, este trabalho teve como objetivo analisar a dependência espacial da fertilidade do solo e sua correlação com a produtividade da soja Glycine max (L) em Dourados - MS.

\section{Material e métodos}

O trabalho foi conduzido no Sitio Rancho Alegre no município de Dourados, MS. O local situa-se em latitude de $22^{\circ} 12^{\prime} \mathrm{S}$, longitude de $54^{\circ} 42^{\prime} \mathrm{W}$ e altitude de 395 $\mathrm{m}$, onde predomina o clima Mesotérmico úmido, do tipo Cwa, segundo a classificação de Köppen, com temperaturas de $20^{\circ}$ a $24^{\circ} \mathrm{C}$ e precipitação de 1250 a $1500 \mathrm{~mm}$ média anual variando (Alvares et al., 2013). Conforme o Sistema Brasileiro de Classificação de Solos (SBCS), o solo da área é um Latossolo Vermelho Distroférrico típico argiloso (Santos et al., 2018). 
Dependência espacial da fertilidade do solo sob plantio direto e suas relações com a produtividade da soja

O mapeamento foi realizado com o GPS de navegação da marca Falker, modelo FieldBox, demarcando o perímetro de 50 ha $(840 \mathrm{~m}$ x $600 \mathrm{~m})$, para geração da malha de amostragem de solo e dos mapas de produti- vidade e fertilidade. A malha utilizada foi quadrada com os pontos no centro, sendo um ponto por hectare (Figura $1)$.

Figura 1 - Área experimental demarcada com os pontos georreferenciados

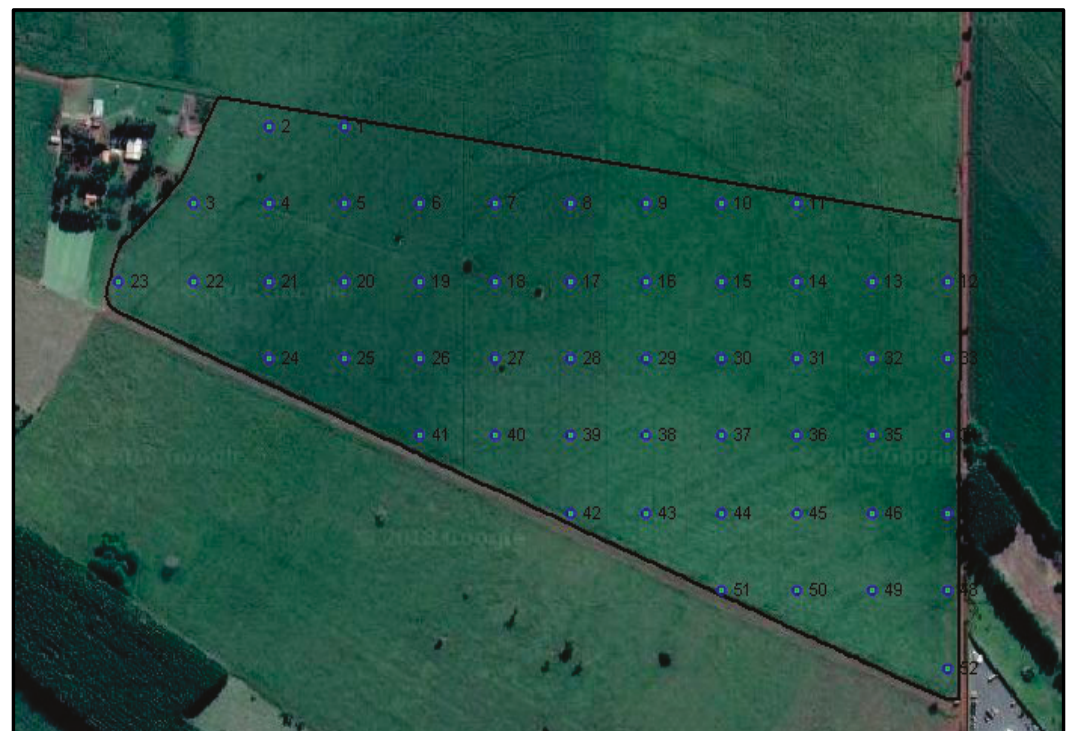

A partir da distribuição dos pontos, realizou-se a coleta de solo na profundidade de 0,00-0,20 m, com seis subamostras utilizando um quadriciclo equipado com amostrador de solo hidráulico automático com broca helicoidal de inox introduzido por sistema hidráulico.

Em seguida, as amostras foram homogeneizadas colocadas em sacos plásticos identificados e submetidas às análises químicas em laboratório. Os atributos avaliados foram: teor de $\mathrm{pH}$ (acidez ativa) do solo, MO (Matéria Orgânica do Solo) ( $\mathrm{g} \mathrm{dm}^{-3}$ ), K (potássio), Ca (Cálcio), Mg (Magnésio), $\mathrm{H}+\mathrm{Al}$ (Acidez Potencial), SB (Soma das Bases), CTC (Capacidade de Troca de Cátios) (mmol dm ${ }^{3}$ ), V (Saturação por Bases) (\%) e P (Fósforo) de acordo com a metodologia de Raij (2001).

Com os dados do laboratório foram gerados os mapas de fertilidade e realizado a correção do solo com calcário $\left(2,118 \mathrm{~kg} \mathrm{ha}^{-1}\right)$, gesso (800 kg ha-1), e cloreto de potássio (200 $\mathrm{kg} \mathrm{ha}^{-1}$ ) a lanço, na superfície do solo, sem incorporação. A seguir foi realizada a semeadura da soja, com uma população de 300.000 plantas ha-1, utilizando a cultivar Brasmax Potência RR, com adubação do formulado N P K 02-20-20 (300 kg ha-1).

Para obtenção dos dados de produtividade, foi realizada a coleta manual da soja em uma área útil de $3,6 \mathrm{~m}^{2}$ (duas linhas de quatro metros espaçadas de 0,45 $\mathrm{m})$. As amostras foram coletadas e identificadas nos 52 pontos georreferenciados, as quais foram determinados e a umidades corrigidas a $13 \%$.

Os dados obtidos foram submetidos a análise de estatística descritiva, sendo analisados os valores médios, mínimo e máximo, distribuição de frequência dos dados, coeficiente de variação (CV) e coeficientes de assimetria e curtose. Para a geração de mapas, parâmetros geoestatítiscos foram gerados, e a validação dos modelos matemáticos por meio de semivariograma e validação cruzada através do programa GS + (Robertson, 1998).

\section{Resultados e discussão}

Os resultados da estatística descrita são apresentados na Tabela 1, na qual pode-se observar que os valores de capacidade de troca de cátions (CTC), acidez potencial $(\mathrm{H}+\mathrm{Al})$, fósforo $(\mathrm{P})$, potássio $(\mathrm{K})$, cálcio $(\mathrm{Ca})$, magnésio $(\mathrm{Mg})$, acidez ativa $(\mathrm{pH} \mathrm{CaCl2})$ saturação por bases (V\%) e matéria orgânica do solo (MO) estão presentes no solo em níveis adequados à produção agrícola, segundo a classe de interpretação utilizada. A relação Cálcio:Magnésio manteve-se em 3:1; desse modo, conforme Malavolta (2006), sendo adequada à cultura.

Os valores para todos os atributos químicos do solo encontram-se em níveis adequados recomendados para cultura da soja. Os teores de K estão na faixa de "muito alto", mas o percentual médio de participação do K na CTC é de 2\%. De acordo com Malavolta (2006), o percentual ideal de K na saturação é entre 3 e 5\%. Portanto os teores de K são adequados, mas o percentual de participação na CTC está abaixo do ideal.

Com base nos coeficientes de correlação entre produtividade de soja e atributos do solo, pode-se concluir que, para os valores de CTC $(-0,30)$ e acidez potencial $(-0,29)$, as correlações foram significativas negativa e, o fósforo $(0,30)$ obteve uma correlação significativa positiva em relação à produtividade da soja. 
Londero, R. et al.

Tabela 1 - Estatística descritiva de atributos químicos do solo no Sítio Ranho Alegre situado na região de Dourados - MS, safra 2017-18

\begin{tabular}{ccccccccc}
\hline Variável & Média & Variância & CV\% & Mínimo & Máximo & Assimetria & Curtose & CR \\
\hline $\mathrm{CTC}^{1}$ & 159,60 & 16,24 & 10,1 & 128 & 187 & $-0,09$ & $-0,92$ & 0,878 \\
$\mathrm{H}^{+} \mathrm{Al}^{1}$ & 54,28 & 45,38 & 12,4 & 37 & 71 & $-0,14$ & 0,34 & 0,915 \\
$\mathrm{P}^{2}$ & 12,01 & 26,56 & 42,8 & 5 & 32 & 1,38 & 3,05 & 0,471 \\
$\mathrm{~K}^{1}$ & 3,34 & 1,11 & 31,5 & 1,9 & 7,5 & 2,03 & 5,23 & 1,094 \\
$\mathrm{Ca}^{1}$ & 76,44 & 218,44 & 19,3 & 42 & 105 & $-0,15$ & $-0,78$ & 0,926 \\
$\mathrm{Mg}^{1}$ & 25,55 & 9,82 & 12,2 & 16 & 31 & $-0,49$ & 0,25 & 0,888 \\
$\mathrm{PH} \mathrm{CaCl}^{2}$ & 5,44 & 0,03 & 3,3 & 5 & 5,9 & $-0,34$ & 0,01 & 0,803 \\
$\mathrm{~V}^{1}$ & 65,53 & 30,84 & 8,4 & 49 & 78 & $-0,51$ & 0,47 & 0,958 \\
$\mathrm{MO}^{3}$ & 37,00 & 17,37 & 11,2 & 28 & 46 & 0,24 & $-0,58$ & 0,246 \\
Produção & 3641 & 290307 & 14,7 & 2415 & 4905 & 0,35 & 0,06 & 0,357 \\
\hline
\end{tabular}

CV: coeficiente de variação; ${ }^{1} \mathrm{mmol} \mathrm{dm}{ }^{-3} ;{ }^{2} \mathrm{mg} \cdot \mathrm{dm}^{-3},{ }^{3} \mathrm{~g} \cdot \mathrm{dm}^{-3},{ }^{4} \mathrm{~kg}$ ha-1 , CR: coeficiente de regressão da validação cruzada.

Segundo Dalchiavon et al. (2012), a variabilidade de um atributo pode ser classificada de acordo com a magnitude de seu coeficiente de variação (CV), em baixo (CV < $10 \%)$, médio $(10 \%<\mathrm{CV}<20 \%)$, alto $(20 \%<$ CV $<30 \%$ ) e muito alto (CV $>30 \%)$. Com base nesta classificação, portanto, observou-se que a maior parte dos atributos químicos apresenta CV médio, exceto o $\mathrm{pH}$ e V\%, com valores baixos entre 3,3 e 8,4\%; e P e K com valores muito altos entre 42,8 e $31,5 \%$.

De acordo com Dalchiavon et al. (2012), trabalhando em Latossolo Vermelho Distroférrico sob plantio direto, a variabilidade dos atributos químicos do solo é consequência de interações complexas em processos de sua formação e de práticas de manejo do solo e da cultura, com impacto principalmente nas camadas superficiais do solo. Todavia, neste estudo, a variabilidade nos atributos da fertilidade do solo, possivelmente, esteve associada ao efeito das sucessivas e irregulares adubações e calagens, que vêm sendo aplicadas na área (Gontijo et al., 2012; Zonta et al., 2014).

Os atributos químicos que apresentaram coeficientes de assimetria positivos foram P 1,38 e o K 2,03. Os demais atributos apresentaram coeficientes de assimetria negativa. O coeficiente de assimetria é utilizado para caracterizar como e quanto a distribuição de frequências se afasta da simetria, sendo que: se coeficiente de assimetria maior que zero tem-se que a distribuição é assimétrica à direita (positiva): se coeficiente de assimetria menor que zero distribuição é assimétrica à esquerda (negativa): e se coeficiente de assimetria igual a zero a distribuição é simétrica (Zanão Júnior et al., 2010).

Os atributos químicos que apresentaram coeficiente de curtose com distribuição mesocúrtica (normal) foi o $\mathrm{pH} \mathrm{CaCl2}$, distribuição platicúrtica (menor que zero) foi o Ca e a CTC e distribuição leptocúrtica (maior que zero) os demais atributos químicos. O coeficiente de curtose é utilizado para caracterizar a forma da distribuição de frequências quanto ao seu achatamento. $O$ termo médio de comparação é a distribuição normal que apresente valor de coeficiente de curtose igual a zero tem-se então a distribuição mesocúrtica, se coeficiente de curtose menor que zero a distribuição será platicúrtica, e se coeficiente de curtose maior que zero tem-se a distribuição leptocúrtica. Sendo que todas as variáveis apresentaram valores próximas de zero.

A dependência espacial dos atributos químicos do solo e produtividade da soja foram analisadas através da análise de semivariogramas (Tabela 2). Os parâmetros avaliados apresentaram forte dependência espacial, exceto a MO que foi moderada. Esta dependência está relacionada com baixo valor do efeito pepita em relação ao patamar. Os semivariogramas constataram que as variáveis $\mathrm{pH} \mathrm{CaCl}_{2}$ e $\mathrm{MO}$ foram ajustadas ao modelo esférico, $\mathrm{P}$ e $\mathrm{H}+\mathrm{Al}$ ao modelo exponencial e os demais ao modelo gaussiano.

Vieira et al. (2010) recomendam que o usuário escolha um destes três modelos segundo o comportamento de seus semivariogramas para distancias menores do que o alcance), faça o ajuste usando algum método de otimização dos parâmetros $\mathrm{C}$, $\mathrm{C} 1$ e a, e submeta este modelo ao processo da validação pelo "jack knifing". Este método, embora trabalhoso, elimina qualquer possibilidade de ajuste inadequado porque os resultados estarão dentro de alguns padrões estatísticos.

Após a escolha do melhor modelo para interpolação, procedeu-se a geração de mapas para todos os atributos (Figura 2).

Os resultados da análise descritiva para a produtividade da cultura de soja na safra 2017/18 estão 
Dependência espacial da fertilidade do solo sob plantio direto e suas relações com a produtividade da soja

apresentados na Figura 3, na qual nota-se que a cultura da soja apresentou padrões de produtividade altamente satisfatórios, obtendo média de $3641,21 \mathrm{~kg} \mathrm{ha}^{-1}$. Ainda assim, constatou-se uma grande variação entre a produtividade mínima, de 2415,00 $\mathrm{kg} \mathrm{ha}^{-1}$ e máxima, de
4905,00 kg ha-1, tendo uma amplitude de 2490,00 kg ha $^{-1}$, significando o mesmo que 41,5 sc ha-1. Resultando em um CV de $14,7 \%$.

Tabela 2 - Parâmetros do semivariogramas ajustados aos dados dos atributos químicos do solo no Sítio Ranho Alegre região de Dourados - MS safra 2017-18

\begin{tabular}{|c|c|c|c|c|c|c|c|}
\hline Variável & Modelo & $\mathrm{CO}$ & $\mathrm{C} 1$ & GDE\% $\%$ & $\mathrm{~A}(\mathrm{~m})$ & R2 & SQR \\
\hline CTC & Gaussiano & 13,00 & 367,7 & 3,4 & 252 & 0,978 & 1294 \\
\hline $\mathrm{H}+\mathrm{Al}$ & Exponencial & 0,40 & 61,40 & 0,6 & 227 & 0,970 & 18,5 \\
\hline $\mathrm{P}$ & Exponencial & 4,82 & 21,34 & 18,4 & 92 & 0,826 & 4,33 \\
\hline K & Gaussiano & 0,32 & 1,753 & 15,4 & 529 & 0,989 & 7,633 \\
\hline $\mathrm{Ca}$ & Gaussiano & 21,80 & 293,4 & 6,90 & 255 & 0,989 & 418 \\
\hline $\mathrm{Mg}$ & Gaussiano & 2,67 & 8,91 & 23,0 & 203 & 0,997 & 0,077 \\
\hline $\mathrm{pH} \mathrm{CaCl}{ }_{2}$ & Esférico & 0,008 & 0,0304 & 21,5 & 420 & 0,968 & 7,402E-06 \\
\hline V\% & Gaussiano & 7,70 & 32,38 & 19,2 & 233 & 0,996 & 1,73 \\
\hline MO & Esférico & 8,23 & 13,72 & 37,4 & 508 & 0,947 & 3,03 \\
\hline Produção & Gaussiano & 30900 & 3020 & 10,2 & 93 & 0,968 & $6,84 \mathrm{E}+07$ \\
\hline
\end{tabular}

C0: efeito pepita; C1: variância estrutural; A: alcance; R2: coeficiente de determinação; GDE: grau de dependência espacial; SQR: Soma de quadrado de resíduo

Figura 2 - Mapa de CTC (A), acidez potencial (B), fósforo (C), potássio (D), cálcio (E), magnésio (F), pH (G), V\% (H) e matéria orgânica (I), coletados em Dourados - MS safra 2017-18

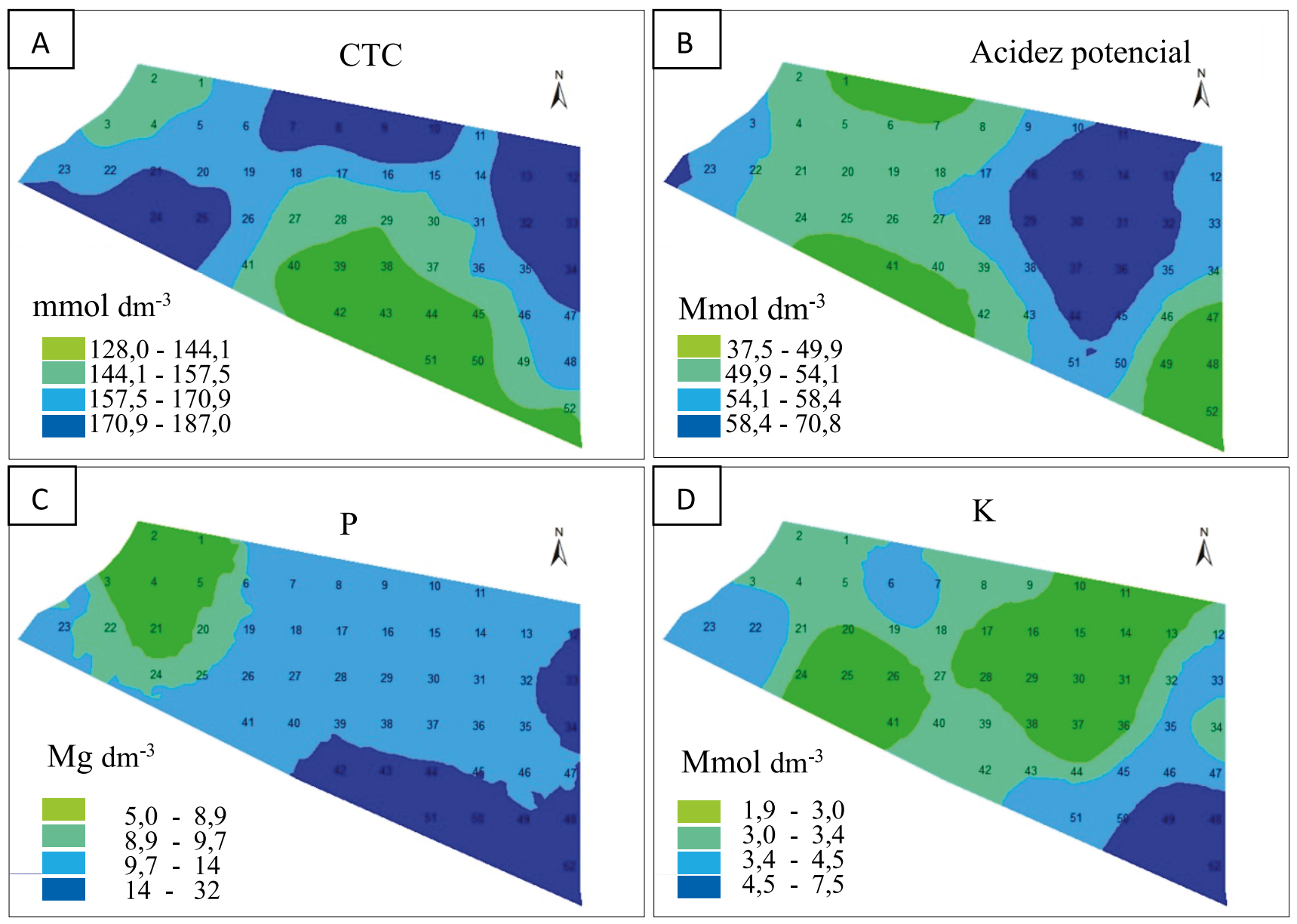

Cad. Ciênc. Agrá., v. 12, p. 01-08, https://doi.org/10.35699/2447-6218.2020.25450 
Londero, R. et al.

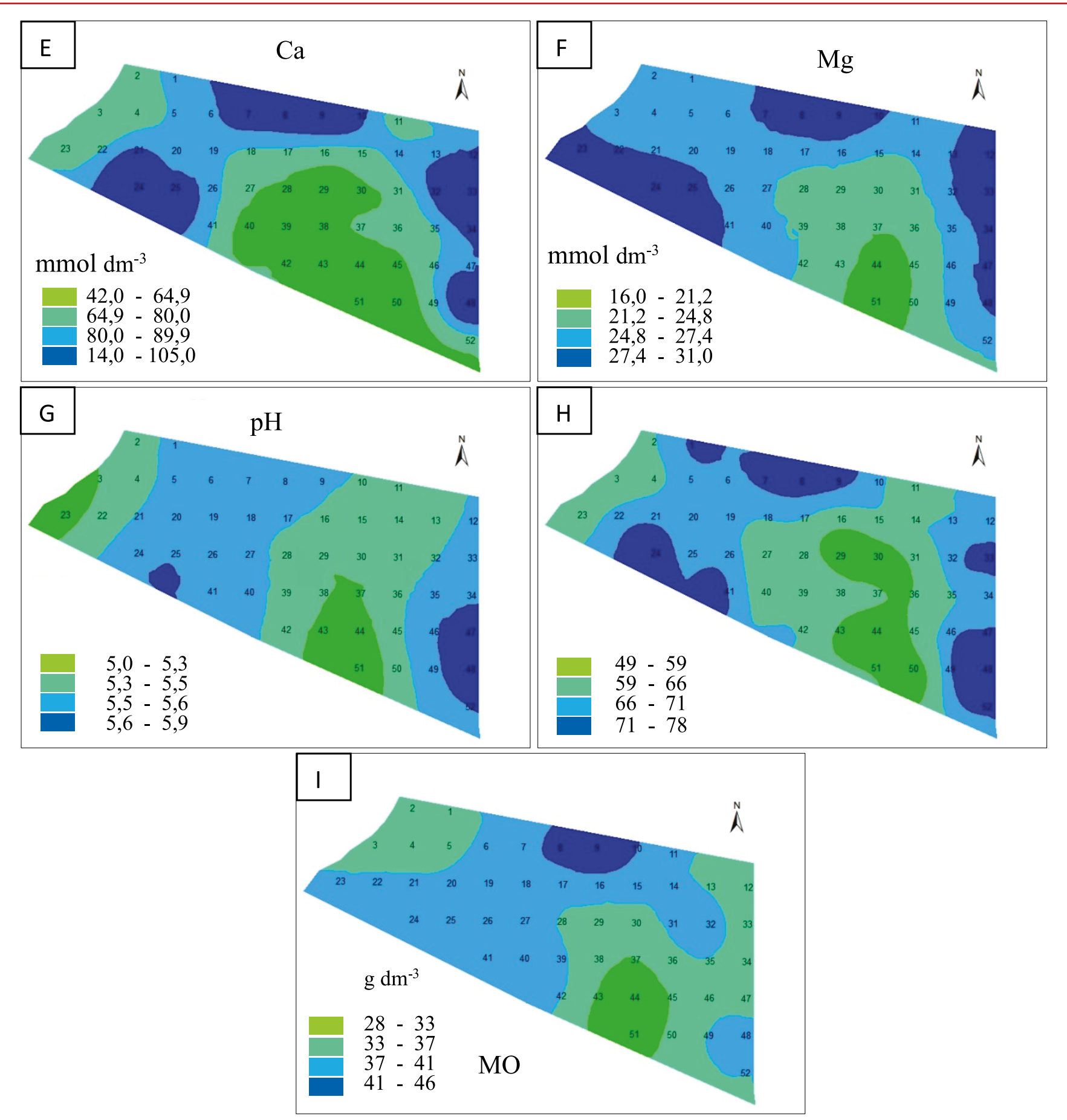

Os valores próximos da média indicam que a produtividade tem distribuição assimétrica positiva, fato confirmado pelo GS +, assim pode-se dizer que a distribuição tende a normal. Com relação à análise geoestatística utilizou-se o critério do menor valor da soma do quadrado dos resíduos (SQR), técnica que melhor possibilita avaliar a dependência espacial da variável. Diante disso, o semivariograma que melhor se ajustou à produtividade da soja foi o modelo gaussiano.

A variável demostrou forte dependência espacial, sendo de 10,2\% para GDE, conforme Cambardella et al. (1994), segundo o qual as variáveis que apresentam forte dependência espacial são mais influenciadas por propriedades intrínsecas do solo.
A importância do alcance (a) para o planejamento e avaliação experimental, sendo capaz de contribuir na definição dos procedimentos amostrais. Determinando como a distância máxima entre amostras onde há dependência espacial, o alcance possivelmente seja o parâmetro mais importante e de aplicação prática da análise geoestatística.

Segundo Gatiboni et al. (2007), em um sistema de plantio direto consolidado por um manejo correto de muitos anos, associado a solos com elevados teores de argila e MO, pode levar o solo a conter uma boa reserva de P. O que leva a uma estabilização do solo pelo plantio direto consolidado influenciando a forte dependência espacial da fertilidade do solo na aérea deste estudo. 
Figura 3 - Mapa de Produtividade de soja em Dourados-MS safra 2017/18

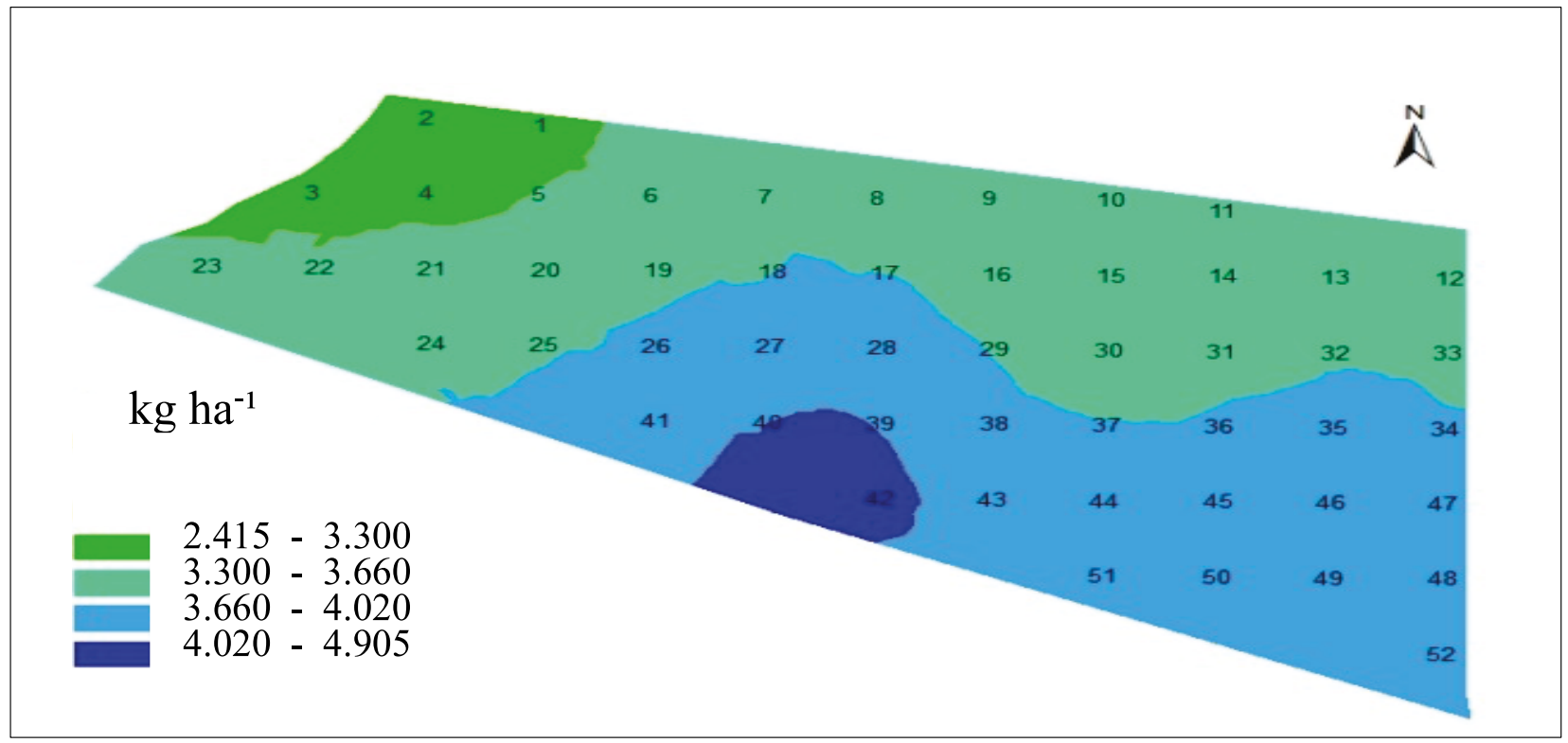

A interpretação do mapa de produtividade é imprescindível para a correção dos fatores de produção que persistem ao longo do tempo, tais como, variação do tipo do solo na área plantada, acidez do solo em locais específicos e deficiência de fertilidade. Segundo Molin et al. (2015), os mapas de produtividade são considerados como a alternativa mais completa para discriminar a variabilidade espacial das lavouras. Sendo assim, elaborou-se o mapa de distribuição espacial da produtividade da cultura a partir dos parâmetros do semivariograma ajustado e a técnica de interpolação dos dados por krigagem.

Com base nos coeficientes de correlação entre produtividade de soja e atributos químicos do solo foi constatado significância para a CTC, acidez potencial e fósforo. A correlação do fósforo $(0,3)$ e CTC $(-0,3)$, significativo a $5 \%$, ou seja, a cada $0,3 \mathrm{mg} \mathrm{dm}^{-3}$ de fósforo e CTC presente no solo, houve um incremento de $1 \mathrm{~kg}$ ha $^{-1}$ na produtividade da soja e a cada $-0,29 \mathrm{mmol} \mathrm{dm}^{-3}$ de acidez potencial houve redução em $1 \mathrm{~kg} \mathrm{ha}^{-1}$ (Tabela $3)$.

Os altos valores de CV para o $\mathrm{P}(42,80 \%)$ são normalmente encontrados em trabalhos com solos tropicais, como o de Dalchiavon et al. (2012), atribuído à dificuldade na adubação fosfatada que sofre interferências de vários fatores, como, por exemplo, o teor de argila. No entanto, apesar da variabilidade verificada para este elemento, a correção do solo se mostrou eficaz para elevar o teor a níveis satisfatórios à cultura o que foi evidenciado pela correlação positiva, possivelmente devido à elevação do pH que minimizou a interação entre os colóides e o íon fosfato, amenizando as reações de adsorção (Dalchiavon et al., 2012).

Tabela 3 - Coeficientes de correlação linear de Pearson da distribuição de atributos químicos do solo e rendimento de grãos no Sítio Ranho Alegre região de Dourados- MS safra 2017-18.

\begin{tabular}{cccc}
\hline Avaliação & Coeficiente correlação & Ajuste R2 & Significância \\
\hline $\mathrm{CTC}$ & $-0,30$ & 2,22 & $0.05^{*}$ \\
$\mathrm{H}+\mathrm{Al}$ & $-0,29$ & 2,11 & $0.05^{*}$ \\
$\mathrm{P}$ & 0,30 & 2,208 & $0,05^{*}$ \\
$\mathrm{~K}$ & 0,25 & 1,832 & $\mathrm{NS}$ \\
$\mathrm{Ca}$ & $-0,20$ & 1,435 & $\mathrm{NS}$ \\
$\mathrm{Mg}$ & $-0,08$ & 0,594 & $\mathrm{NS}$ \\
$\mathrm{pH} \mathrm{CaCl}$ & 0,02 & 0.189 & NS \\
$\mathrm{V} \%$ & 0,026 & 0,188 & NS \\
$\mathrm{MO}$ & 0,018 & 0,25 & NS
\end{tabular}

*Correlação significativa $\mathrm{p}<0,05$ pelo Teste T; NS: não significativo. 
Londero, R. et al.

\section{Conclusão}

Os atributos químicos do solo, exceto a matéria orgânica do solo, e a produtividade da soja apresentaram forte dependência espacial, validando os mapas temáticos.
Houve correlação positiva entre fósforo e produtividade da soja, enquanto que a correlação entre $\mathrm{H}+\mathrm{Al}$, CTC e produtividade da soja foi negativa.

\section{Referências}

Alvares, C. A.; Stape, J. L.; Sentelhas, P. C.; Gonçalves, M.; Leonardo, J.; Spavorek, G. 2013. Köppen's climate classification map for Brazil. Meteorologische Zeitschrift, 22:711-728. Doi: 10.1127/09412948/2013/0507.

Brasil. 2017. Ministério da Agricultura, Pecuária e Abastecimento MAPA. Agricultura de precisão 19. Brasília, 2017. 15p. Disponível em: <http://www.agricultura.gov.br/assuntos/ camaras-setoriais-tematicas/ documentos/camaras-setoriais/florestas-plantadas/anos-anteriores/ agricultura-de-precisao-19.pdf/view>. Acesso em: 23 set. 2019.

Cambardella CA, Moorman JM, Novak TB, Karlen DL, Turco RF, Konopka AE (1994) Field-scale variability of soilproperties in Central Iowa Soils. Soil Science Society of America Journal 58(5):1501-1511.

COMPANHIA NACIONAL DE ABASTECIMENTO - CONAB. 2019. Boletim da Safra de Grãos: 1o Levantamento - Safra 2019/20 - Tabela de levantamento. Brasília: CONAB, 2019. Disponível em: < https:// www.conab.gov.br/info-agro/safras/graos > . Acesso em: 13 out. 2019.

Dalchiavon, F. C.; Carvalho, M. P; Andreotti, M.; Montanari R. 2012. Variabilidade espacial de atributos da fertilidade de um Latossolo Vermelho Distroférrico sob Sistema Plantio Direto. Revista Ciência Agronômica, 43: 453-461.

Freitas, R. E.; Mendonça, M. A. A. 2016. Expansão Agrícola no Brasil e a Participação da Soja: 20 anos. Revista de Economia e Sociologia Rural, 54:497-516. Doi: http://dx.doi.org/10.1590/1234-5678180694790540306 .

Gatiboni, L. C.; Kminski, J.; Rheinheimer, D. S.; Flores, J. P. C. 2007. Biodisponibilidade de formas de fósforo acumuladas em solo sob sistema plantio direto. Revista Brasileira de Ciência do Solo, 31: 691-699. Doi: 10.1590/S0100-06832007000400010.

Gontijo, I.; Nicole, L. R.; Partelli, F. L.; Bonomo, R.; Santos, E.O.J. 2012. Variabilidade e correlação espacial de micronutrientes e matéria orgânica do solo com a produtividade da pimenta-do-reino. Revista Brasileira de Ciência do Solo 36(4):1093-1102. Doi: 10.1590/S010006832012000400004.

Grego, C. R.; Vieira, S. R. 2005. Variabilidade espacial de propriedades físicas do solo em uma parcela experimental. Revista Brasileira de Ciência do Solo, 29(2):169-177. Doi: https://doi.org/10.1590/S010006832005000200002 .

Malavolta, E. 2006. Manual de nutrição mineral de plantas. São Paulo: Agronômica Ceres, 2006. 638p.

Molin, J. P.; Amaral, L. R.; Colaço, A. F. 2015. Agricultura de precisão. 1. ed. Oficina de textos. São Paulo.
Raij, B. Van et al. (ed) Análise quimica para avaliação da fertilidade de solos tropicais. Campinas: Instituto Agronômico de Campinas, 2001. 212 p.

Resende, A. V.; Hurtado, S. M. C.; Vilela, M. F.; Corazza, E. J.; Shiratsuchi, L. S. 2004. Aplicações da agricultura de precisão em sistemas de produção de grãos no Brasil. In: Sousa, D. M. G.; Lobato, E., 2014. Cerrado correção do solo e adubação.

Robertson, G.P. 1998. GS+: Geostatistics for the Environmental Sciences. Plainwel: Gamma Design Software.

Santos, E. O. J.; Gontijo, I.; Silva, M.B.; Drumond Neto, A.P. 2015. Variabilidade espacial de macronutrientes em uma lavoura de café conilon no Norte do Espírito Santo. Revista Ciência Agronômica, 46(3):469-476.

Santos, H. G.; Jacomine, P. K. T.; Anjos, L. H. C.; Oliveira, V. A.; Lumbreras, J. F.; Coelho, M. R.; Almeida, J. A.; Araujo Filho, J. C.; Oliveira, J. B.; Cunha, T. J. 2018. Sistema Brasileiro de Classificação de Solos. 5. ed. Brasília: Embrapa, 187 p. Disponível em: https:// www.embrapa.br/solos/busca-de-publicacoes/ /publicacao/1094003/ sistema-brasileirode-classificacao-de-solos. Acesso em: 18 out. 2018.

Santos, R. O.; Franco, L. B.; Silva, S. A.; Sodré, G. A.; Menezes, A. A. 2017. Spatial variability of soil fertility and its relation of cocoa yield. Revista Brasileira de Engenharia Agrícola e Ambiental, 21:88-93. Doi: https://doi.org/10.1590/1807-1929/agriambi.v21n2p88-93.

Soares Filho, R.; Cunha, J. P. A. R. 2015. Agricultura de precisão: particularidades de sua adoção no sudoeste de Goiás - Brasil. Engenharia Agricola, 35(4):689-698. Doi: 10.1590/1809-4430-Eng. Agric.v35n4p689-698/2015.

Trentin, R. G.; Modolo, A. J.; Vargas, T. O.; Campos, J. R. R.; Adami, P. F; Baesso, M. M. 2018. Soybean productivity in Rhodic Hapludox compacted by the action of furrow openers. Acta Scientiarum. Agronomy 40:1-9. Doi: http://dx.doi.org/10.4025/actasciagron.v40i1.35015.

Vieira, S. R.; Carvalho, J. R. P.; Paz Gonzalez, A. 2010. Jack knifing for semivariogram validation. Bragantia, 69: 97-105. Doi: http://dx.doi. org/10.1590/S0006-87052010000500011.

Zanão Júnior, L. A.; Lana, R. M. Q.; Carvalho-Zanão, M. P.; Guimarães, E. C. 2010. Variabilidade espacial de atributos químicos em diferentes profundidades em um Latossolo em sistema de plantio direto. Revista Ceres, 57:429-438. Doi: https://doi.org/10.1590/S0034737X2010000300021.

Zonta, J. H.; Brandão, Z. N.; Medeiros, J. C.; Sana, R. S.; Sofiatti, V. 2014. Variabilidade espacial da fertilidade do solo em área cultivada com algodoeiro no Cerrado do Brasil. Revista Brasileira de Engenharia Agrícola e Ambiental, 18(6):595-602. Doi: 10.1590/S1415-43662014000600005. 